\title{
An update on hyper-acute management of ischaemic stroke
}

\author{
Authors: Ajay Bhalla, ${ }^{A}$ Mehool Patel ${ }^{B}$ and Jonathan Birns ${ }^{C}$
}

This article aims to provide a comprehensive overview of key advances on various aspects of hyper-acute management of acute ischaemic stroke. These include neuroimaging, acute stroke unit care, management of blood pressure, reperfusion therapy including intravenous thrombolysis, mechanical thrombectomy and decompressive hemicraniectomy for malignant stroke syndrome. The challenge ahead is to ensure these evidence-based treatments are now being delivered and implemented to maximise the benefits across the population.

KEYWORDS: management, ischaemic stroke, thrombolysis, thrombectomy

\section{DOI: 10.7861/clinmed.2020-0998}

\section{Introduction}

Ischaemic stroke is a common emergency presentation to hospital with improving survival rates owing to access to specialist organised stroke care. There has been considerable advancement in hyper-acute stroke treatments in the last decade, which has resulted in improved outcomes and revolutionised acute stroke care from a disease with no treatment to one with multiple proven options. ${ }^{1}$ The premise for acute stroke care is to salvage viable ischaemic brain tissue (ischaemic penumbra) surrounding the irreversibly injured core through reperfusion. ${ }^{2}$ This article provides a comprehensive update on contemporary evidence-based management of acute stroke.

\section{Neuroimaging for ischaemic stroke}

Computed tomography (CT) of the brain using the 10-point Alberta Stroke Program Early CT Score (ASPECTS) is a useful modality in denoting early ischaemic changes. ${ }^{3}$ Although the hyper-dense artery sign is common feature of large vessel occlusion, it cannot be identified in up to $50 \%$ of acute middle cerebral artery (MCA) occlusions using non-contrast CT (Fig 1).

Authors: ${ }^{\text {A }}$ consultant in stroke medicine, St Thomas' Hospital, London, UK; ${ }^{B}$ consultant in stroke medicine, geriatrics and general medicine, University Hospital Lewisham, Lewisham, UK; ${ }^{C}$ consultant in stroke medicine, geriatrics and general medicine, St Thomas' Hospital, London, UK and deputy head of School of Medicine, Health Education England, London, UK
CT angiography (CTA) and CT perfusion (CTP) is important in identifying large vessel occlusion, collateral circulation and salvageable tissue for reperfusion interventions. Colour-coded CTP maps can identify brain regions with mismatch gaps by comparing reductions in cerebral blood flow (CBF) with regions of significant hypoperfusion, as reflected by delays in contrast arrival times (Tmax delays; Fig 2). 4,5 Magnetic resonance imaging (MRI), which has greater sensitivity in detecting ischaemia than $\mathrm{CT}$, can also be used to assess salvageable brain tissue through magnetic resonance diffusion and perfusion maps. ${ }^{7}$

\section{Acute stroke unit care}

Provision of stroke unit care is the single most effective intervention for all stroke patients. Stroke units are associated

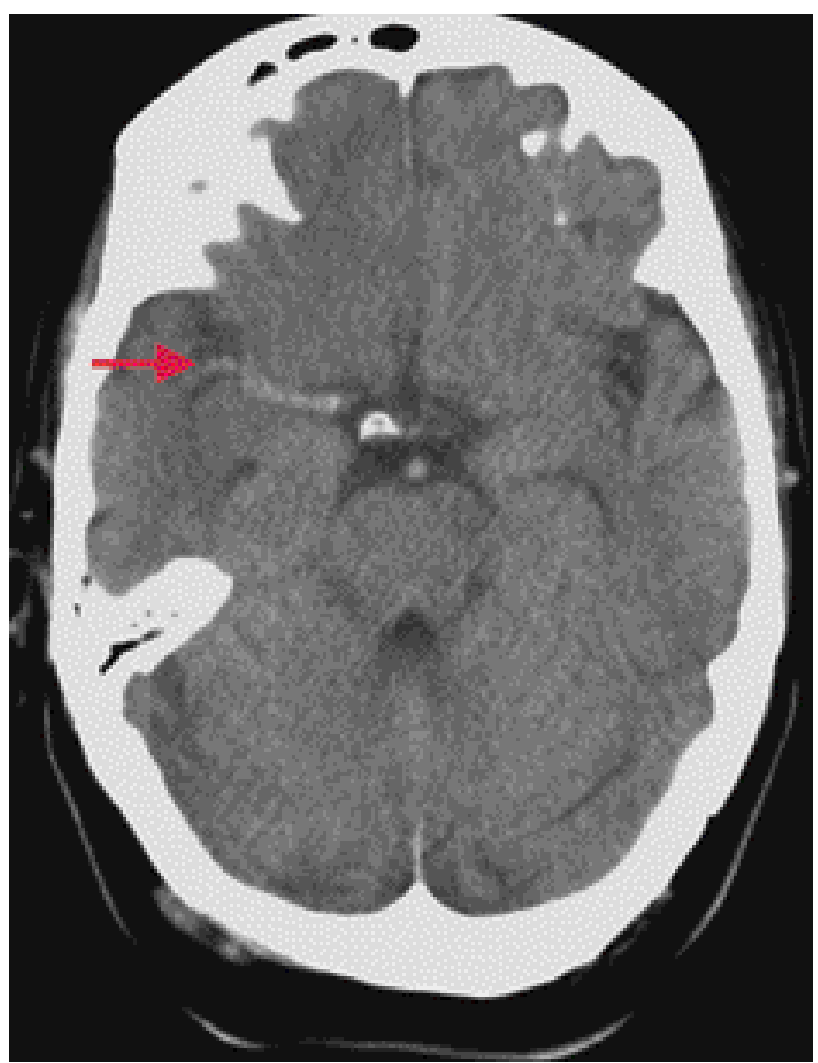

Fig 1. Computed tomography of the brain demonstrating hyper-dense middle cerebral artery sign (arrow). 
Fig 2. Computed tomography perfusion demonstrating cerebral blood flow (CBF) $<30 \%$ (ischaemic core) and time to maximal delay (Tmax $>6$ seconds) in a patient with an acute right middle cerebral artery occlusion. Difference between CBF (pink) and Tmax (green) indicating a target mismatch volume of $65 \mathrm{~mL}$ ( $46 \mathrm{~mL}-111 \mathrm{~mL}$; ratio 2.4), highlighting indication for reperfusion with mechanical thrombectomy. Reproduced with permission from Laughlin B, Cahn A, Tai WA, Moftakhar P. RAPID automated CT perfusion in clinical practice. Pract Neurol 2019;2019:41-55. ${ }^{6}$

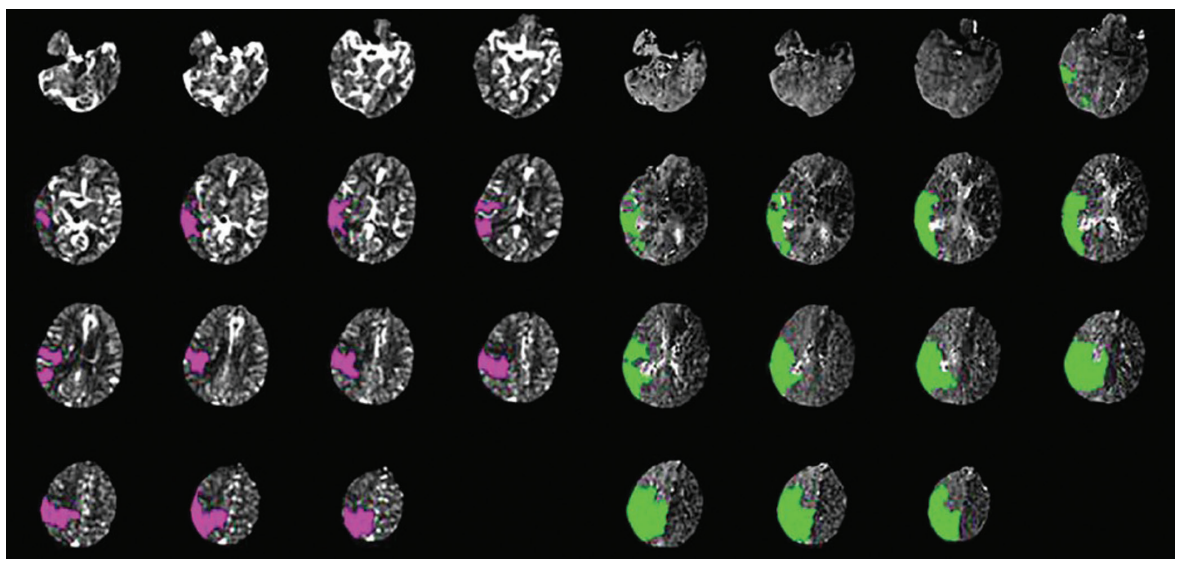

with reduced death or dependency (odds ratio (OR) 0.75; 95\% confidence interval (CI) $0.66-0.85$ ) facilitated by stroke multidisciplinary care. ${ }^{8}$ A key function for stroke unit care is to limit neurological deterioration by monitoring for and correcting abnormal physiological parameters. ${ }^{9-11}$ This includes strategies to correct hypotension, hypertension, hyperglycaemia, hypoxia, pyrexia, dehydration and positioning (Table 1), and to optimise management of nutrition and continence. ${ }^{9,10,12,13}$ Training staff in the use of standardised protocols to manage physiological status can significantly improve outcomes. ${ }^{13,22}$

\section{Blood pressure management}

In acute stroke, there is an inherent tendency for the blood pressure (BP) to be high due to disruption of cerebral autoregulation. ${ }^{23}$ Various strategies and agents to manage $\mathrm{BP}$ in acute stroke have been examined. A study examining transdermal glyceryl trinitrate (GTN) patches showed that while BP was lowered, this did not improve functional outcome. ${ }^{24}$ An ambulance-based randomised trial examining the use of transdermal GTN in acute stroke that was then continued in hospital for 4 days showed that pre-hospital treatment with GTN did not improve functional outcome. ${ }^{25}$ Another study also showed that immediate BP reduction in non-thrombolysed ischaemic stroke patients within 48 hours did not reduce the likelihood of death and major disability at 14 days. This study aimed at lowering systolic BP by $10-25 \%$ within the first 24 hours after randomisation, achieving BP levels of less than $140 / 90 \mathrm{mmHg}$ within 7 days, and maintaining this level during hospitalisation. ${ }^{26}$ For patients receiving thrombolysis with

Table 1. Specific management of physiological parameters ${ }^{13,14}$

\section{Physiological parameter Management guidance}

Oxygenation

Hydration

Temperature

Blood pressure

Blood glucose

Venous thromboembolism

Head positioning
Supplemental oxygen only if oxygen saturation is below $95 \%$ and there is no contraindication ${ }^{15}$ Hyperbaric oxygen is not recommended unless stroke is caused by air embolisation

Regularly assess and ensure adequate oral/intravenous replacement so that normal hydration is maintained

Sources of hyperthermia (temperature $>38^{\circ} \mathrm{C}$ ) should be identified and treated, and antipyretic medications should be administered to lower temperature

Benefit of treatment with induced hypothermia is uncertain so this is not routinely recommended

Patients with stroke should only receive blood-pressure-lowering treatment if there is an indication for emergency treatment, such as systolic blood pressure $>185 \mathrm{mmHg}$ or diastolic blood pressure $>110$ $\mathrm{mmHg}$ when the patient is otherwise eligible for thrombolysis; hypertensive encephalopathy, hypertensive nephropathy, hypertensive cardiac failure or myocardial infarction; aortic dissection; pre-eclampsia or eclampsia Patients already on anti-hypertensive medication should resume oral treatment once they are medically stable Maintain blood glucose between 5-15 mmol/L, with close monitoring to avoid hypoglycaemia ${ }^{16}$

Immobile patients should be offered intermittent pneumatic compression within 3 days for prevention of deep vein thrombosis, continued for 30 days or until the patient is mobile or discharged, whichever is sooner $^{17}$

It is not advisable for stroke patients to be routinely be given low molecular weight heparin or graduated compression stockings (either full-length or below-knee) for the prevention of deep vein thrombosis ${ }^{18-20}$

An individualised approach should be adopted when comparing lying flat position or head elevation $>30$ degrees in the first 24 hours $^{21}$ 
alteplase, the ENCHANTED trial showed that intensive BP lowering (systolic BP 130-140 mm Hg within 1 hour) reduced intracranial haemorrhage ( $\mathrm{ICH}$ ), but this did not result in improved functional status at 90 days. ${ }^{27}$ Data suggest that excessive BP lowering may worsen cerebral ischaemia and probably results in worse outcome, particularly if there is associated large vessel occlusion. ${ }^{14}$ Evidence also exists that BP variability results in infarct growth and worsens outcome. ${ }^{28} \mathrm{~A}$ Cochrane systematic review on interventions for altering $\mathrm{BP}$ in acute stroke showed insufficient evidence that lowering BP in acute stroke improves functional outcome, and suggested that further trials are needed to identify who would benefit from early treatment. ${ }^{29}$

Current UK guidelines therefore suggest that patients with acute ischaemic stroke should only receive BP lowering treatment if there is an indication for emergency treatment, such as systolic $\mathrm{BP}>185 \mathrm{mmHg}$ or diastolic $\mathrm{BP}>110 \mathrm{mmHg}$ when the patient is otherwise eligible for treatment with alteplase, or hypertensive encephalopathy, nephropathy, cardiac failure or aortic dissection. 13,14 American Heart Association guidelines suggest that a cautious BP reduction by $15 \%$ within the first 24 hours may be reasonable. Patients already on anti-hypertensive medication should resume oral treatment once they are medically stable. 13,14

There are various parenteral options recommended in managing hypertension (BP > 185/110 mmHg) in hyperacute ischaemic strokes that would otherwise be eligible for reperfusion therapy, including labetalol, nicardipine, clevidipine, hydralazine and enalaprilat; if still not controlled, sodium nitroprusside may be considered. ${ }^{14}$ Different treatment options may be appropriate in patients with other comorbidities including acute coronary event, acute heart failure, aortic dissection or pre-eclampsia/eclampsia. ${ }^{14}$

\section{Reperfusion therapy for acute ischaemic stroke}

The key factors in determining whether ischaemia will lead to infarction are the presence and extent of collateral circulation and the time at which recanalisation takes place within the ischaemic penumbra. There are two modalities of reperfusion therapy available: intravenous thrombolysis (IVT) and mechanical thrombectomy (MT).

\section{Intravenous thrombolysis}

Current guidelines recommend the use of IVT with alteplase $(0.9 \mathrm{mg} / \mathrm{kg})$ if treatment is rapidly delivered within 4.5 hours of symptom onset, provided ICH has been appropriately excluded and delivery occurs within the context of an organised stroke service with skilled and trained staff to monitor for complications. ${ }^{13}$ Meta-analysis of individual patient data $(6,756$ patients from nine randomised controlled trials (RCTs)) involving alteplase demonstrated that the number needed to treat (NNT) to achieve a good outcome was 10 for treatment delivered in $\leq 3$ hours, 19 for $3-4.5$ hours and 50 for $>4.5$ hours. The symptomatic ICH rates were $6.8 \%$ vs $1.3 \%$ in the control group, equating to the numbers needed to harm being $18 .{ }^{30}$ There was no significant difference in mortality at 90 days $(17.9 \%$ alteplase vs $16.5 \%$ control). The benefits were observed irrespective of age and stroke severity, highlighting that earlier treatment produces larger proportional benefits. It should be noted that for the 3-4.5 hour subgroup, caution needs to be applied, given that for each patient in whom treatment results in a good outcome (NNT 19), one has a symptomatic ICH; therefore the priority should be to deliver treatment as quickly as possible. The risks of fatal ICH are not insignificant (2\%) with IVT and lower doses of alteplase $(0.6$ $\mathrm{mg} / \mathrm{kg}$ ) have been shown to reduce haemorrhage risk and early mortality but do not deliver equivalent efficacy to conventional doses $(0.9 \mathrm{mg} / \mathrm{kg}){ }^{27}$ Lower doses therefore may be considered by the clinician in order to forgo the benefit of reducing disability in patients who are deemed high risk of early ICH. For patients with very mild measurable neurological deficits, although trial evidence is lacking, the use of thrombolysis may be supported in individual cases if the deficit is deemed to be disabling to the patient. ${ }^{31}$

\section{Wake-up stroke}

$15-25 \%$ of stroke patients will not have a recognised time of onset of stroke, with patients frequently waking from sleep. Several groups have used the concept of a diffusion-weighted imaging / fluid-attenuated inversion recovery (DWI/FLAIR) mismatch (positive DWI lesion but negative FLAIR, indicating that tissue is ischaemic and salvageable rather than infarcted and non-salvageable) to guide thrombolysis. This imaging concept demonstrated a high degree of sensitivity and specificity in predicting onset of stoke within 4.5 hours. ${ }^{32,33}$ The WAKE-UP study tested the efficacy and safety of alteplase in MRI-guided thrombolysis in patients with stroke of unknown time of onset ( $90 \%$ of which were wake-up stroke) using the concept of mismatch. ${ }^{34}$ There was an $11 \%$ difference in favourable outcome in preference to the alteplase group with a non-significant difference in symptomatic ICH ( $2 \%$ alteplase vs $0.4 \%$ placebo). The NNT to afford favourable outcome in this trial was nine patients, highlighting potential expansion of the ischaemic stroke population eligible for recanalisation therapy.

\section{Extending thrombolysis to 4.5-9 hours}

Meta-analysis of individual patient data from three trials (EXTEND, ECASS-4 and EPITHET) has examined the merits of extending thrombolysis with alteplase to 4.5-9 hours including wake-up stroke ( 9 hours from mid-point of sleep) using perfusion imaging to identify salvageable tissue. ${ }^{35}$ Two-thirds of the patients had large vessel occlusion (but did not undergo MT) and $50 \%$ of patients had wake-up stroke. The odds of excellent functional outcome at 90 days were 1.86 (95\% CI 1.15-2.99) in favour of alteplase treatment and this was consistent across age, time window (4.5-6 hours, 6-9 hours and wake-up) as well as in the presence of large vessel occlusion. There were no significant differences in mortality at 90 days (alteplase 14\% vs control $9 \%$ ). A further recent meta-analysis of individual patient data involving alteplase for stroke with unknown time of onset guided by advanced imaging (WAKE UP, EXTEND, THAWS and ECASS 4) showed an absolute $8 \%$ improvement in functional independence despite an increase in symptomatic ICH ( $3 \%$ vs $<1 \%$ in control arm). ${ }^{36}$ These data suggest that IVT may be useful in bridging therapy in conjunction with MT within this specified time frame as well as being considered as stand-alone therapy in the absence of large vessel occlusion. The combination of IVT and MT in an extended time window (4.5-24 hours) is being tested in the TIMELESS trial using tenecteplase. ${ }^{37}$

\section{Alternative agents}

While alteplase is the only licensed thrombolytic Food and Drug Administration agent for ischaemic stroke, tenecteplase has 
potential advantages over the former agent in having greater fibrin sensitivity (and hence being less haemorrhage-inducing), a longer half-life (can be given as a bolus with quicker door-tocompletion time of thrombolysis) and lower costs. There have been five RCTs comparing tenecteplase with alteplase with varying results, with tenecteplase having been shown to be at least effective as alteplase for neurological improvement. ${ }^{38}$ Campbell et al demonstrated that tenecteplase $0.25 \mathrm{mg} / \mathrm{kg}$ compared with standard dose of alteplase $0.9 \mathrm{mg} / \mathrm{kg}$ delivered $<4.5$ hours prior to MT achieved greater recanalisation and improved neurological recovery at 90 days. ${ }^{39}$ Overall, it appeared that lower doses of tenecteplase $(0.25 \mathrm{mg} / \mathrm{kg}$ ) achieved a lower trend of symptomatic haemorrhage rates compared with alteplase, but higher rates of haemorrhage were observed with higher doses of tenecteplase at $0.4 \mathrm{mg} / \mathrm{kg}$. Further studies involving tenecteplase include ATTEST 2 (tenecteplase vs. alteplase $<4.5$ hours), TASTE (tenecteplase vs alteplase with imaging mismatch), TWIST (tenecteplase in wakeup stroke) and TEMPO-2 (tenecteplase in minor with large vessel occlusion). ${ }^{40}$ Therapies such as desmoteplase, argatroban, Gb IIb/ IIIa inhibitors and sono-thrombolysis to augment recanalisation in conjunction with IVT have failed to improve outcomes consistently. ${ }^{41}$

\section{Limitations of thrombolysis}

Large vessel occlusions involving internal carotid artery and proximal MCA tend to only re-canalise between 10-25\% respectively post-IVT, with evidence of residual thrombus angiographically. Several characteristics of thrombus may negate the effects of IVT, such as long thrombus length ( $>8 \mathrm{~mm}$ ), greater thrombus age, the thrombus being platelet- and fibrin-rich rather than of red cell composition, and calcific thrombus material. ${ }^{42}$

\section{Service delivery}

Hospitals with higher volume thrombolysis activity achieve significantly shorter door-to-needle times in administering IVT. ${ }^{43}$ Although using tele-stroke health applications and enhanced paramedic assessments are a potentially attractive model in facilitating stroke diagnosis and delivery of IVT, further developments in these models are required. Mobile stroke units (incorporating an ambulance equipped with an imaging system, a point-of-care laboratory, a telemedicine connection to hospital, and appropriate medication) have been developed with the potential to provide physicians with the necessary information and resources to screen patients safely for IVT eligibility and even initiate thrombolysis 'in the field'.

\section{Mechanical thrombectomy}

There is overwhelming evidence from RCTs for the effectiveness of MT in improving functional outcome at 90 days in patients presenting with proximal occlusion of a large vessel artery in the anterior circulation. Earlier trials failed to demonstrate efficacy due to use of older devices and not deploying uniform protocols for identifying large vessel occlusions, whereas more recent trials used modern devices such as stent retrievers and aspiration devices and placed patient selection under greater scrutiny. ${ }^{44,45}$ The HERMES meta-analysis of individual patient data from five RCTs demonstrated benefit if MT was delivered within 12 hours of onset. The NNT to afford functional independence was between 3.2 and
7.4 patients when compared with best medical treatment. ${ }^{46}$ There were similar rates of symptomatic ICH $(4.4 \%)$ and a trend towards lower mortality (15.3\%). The intra-arterial strategies examined were different in the trials to date, with subtle variations including simple imaging such as CT and CTA (MR CLEAN, ${ }^{47}$ PISTE), ${ }^{48}$ waiting for IVT response before proceeding (MR CLEAN) ${ }^{47}$ and complex imaging such as CTP, MRI and multiphase collaterals with favourable imaging profile (ESCAPE, ${ }^{49}$ EXTEND IA, ${ }^{50}$ SWIFT PRIME, ${ }^{51}$ THRACE, ${ }^{52}$ THERAPY, ${ }^{53}$ and REVASCAT). ${ }^{54}$

For trials delivering MT predominately within 6 hours, which included patients who also received IVT (within 4.5 hours), the rate of functional independence surpassed $60 \%$ using modern stent retriever devices. ${ }^{48,50-52,55}$ Trials involving selective advanced imaging to identify salvageable ischaemic brain tissue also demonstrated good functional outcome ranging from $44-70 \%$ with MT. ${ }^{49-52,56}$ Overall good functional outcome at 90 days was $20 \%$ greater (absolute benefit) with MT compared with best medical therapy.

The mantra of 'time is brain' is as important for MT as it is with IVT, with greater benefits observed if delivered within 4.5 hours of onset and with good collateral circulation. For every hour delay in MT, there is a reduction in reperfusion by $20 \% .{ }^{57}$ UK guidelines have endorsed the use of MT to be delivered as soon as possible in patients with a measurable neurological deficit (National Institutes of Health Stroke Scale (NIHSS) $\geq 6$ ), with the procedure commencing within 5 hours of symptom onset in combination with IVT in confirmed occlusion of the proximal anterior circulation. ${ }^{13,58}$ In addition to this, MT can be used within the same timeframe and criteria when IVT is also contraindicated. Patients included should only have mild disability prior to their stroke.

For those who present within 24 hours, including those with wake-up stroke, there is increasing evidence for MT using perfusion based imaging techniques, with the DAWN trial ${ }^{56}$ looking at patients presenting at between 6-24 hours and the DEFUSE 3 trial $^{59}$ at presentations within 6-16 hours. Absolute benefits (good functional outcome) for patients in the DAWN trial compared with standard medical care equated to $36 \%$, with DEFUSE 3 showing $28 \%$. Accordingly, the NICE 2019 Stroke Guidelines recommends intervention within up to 24 hours if there is salvageable brain tissue ('penumbra') demonstrated by either CTP or DWI MRI sequences. There is also increasing data now supporting the use of MT in patients with M2 occlusions (first division of the MCA). ${ }^{60}$ However, the evidence base for intervening for posterior circulation stroke, including proximal basilar artery occlusion, within $<6$ hours of onset is not robust, with the results of the BASICS trial demonstrating no significant benefit of thrombectomy, but intervention with MT may be considered up to 24 hours in selected cases until further trial evidence is available. ${ }^{61}$

Currently $1.8 \%$ of the stroke population undergoes MT in the UK, with a planned target of $10 \%$ by 2022 for England, Northern Ireland and Wales, equating to 8,000 patients per year. Current estimates suggest that $11-12 \%(10,000-11,500$ stroke admissions) of the UK population would be potentially eligible for MT including additional support from advanced imaging, impacting those who present late (12-24 hours). ${ }^{62}$ At present, studies have consistently demonstrated that MT is likely to be costeffective, with net savings in lifetime analyses. ${ }^{63}$

Facilities for MT are not universally available and there is a need to determine how many specialist centres will be required to ensure maximum geographical provision. The choice of model will depend on local and regional service organisation, 
patient characteristics and volume of admissions. ${ }^{62,64}$ There is also a shortage of trained specialist staff to deliver MT through interventional neuroradiology, and, as such, there is an urgent need to expand this workforce as well as offering credential training for other specialties to support delivery and maintain the necessary skills and expertise for MT. ${ }^{65}$

There are still areas of uncertainty, which include whether patients with large vessel occlusion should forgo IVT and undergo MT directly in order to facilitate timely reperfusion. The Direct MT study suggested that MT alone was non-inferior to a combination of IVT/MT regarding functional outcome, although this needs to be replicated in future trials. ${ }^{66}$ Other areas that require further investigation include the use of alternative thrombolytic agents to alteplase, such as tenecteplase, in combination with MT, establishing the degree of collateral blood flow with advanced imaging, ${ }^{67}$ the optimal anaesthetic strategy (local versus general anaesthetic) ${ }^{68}$ and which devices should be used (stent retrievers vs aspiration devices). ${ }^{69}$

\section{Decompressive hemicraniectomy for malignant stroke syndrome}

Large, space-occupying, hemispheric, ischaemic brain infarcts (described as malignant infarcts) are associated with the development of massive brain oedema, which may lead to herniation and early death. While conservative therapies are not supported by evidence from clinical trials, decompressive hemicraniectomy has been shown to prevent fatal internal displacement of brain tissue (and subsequent herniation) in cases of malignant MCA infarction within 48 hours of stroke, with a potential trigger for such consideration being a decline in the level of consciousness. ${ }^{70}$ Meta-analysis of seven RCTs, involving 338 patients, demonstrated a significant 12 -month $39 \%$ reduction in death $(30 \%$ vs $69 \%$ ) and $13 \%$ increase in the number of patients with mild to moderate disability ( $27 \%$ vs $14 \%) .{ }^{71}$ The distribution of death and disability was similar in trials with additional 6-month and 36-month follow-up data. ${ }^{71}$ Studies have suggested that earlier surgery is associated with lower mortality rates and better functional outcome. ${ }^{72}$ While early trials excluded patients over the age of 60 , more recently, the benefit of hemicraniectomy has been validated in older patients. ${ }^{73}$ While decompressive hemicraniectomy has a NNT of 2 in terms of reducing mortality, treatment decisions need to focus on the acceptable quality of life after surgery. ${ }^{73}$ In addition to surgical decompression for malignant MCA infarction, ventriculostomy and suboccipital decompressive craniectomy has been shown to be effective in the setting of hydrocephalus complicating acute cerebellar infarction. $^{74}$

\section{Future directions}

Huge strides have been made in acute ischaemic stroke management in recent years. The challenge of how to translate improvements in cerebral perfusion from acute interventions into functional outcomes remains. Close attention to therapy assessments and interventions alongside medical treatments aligned to quality improvement strategies is an important area for the future, and uptake of technological advances, such as the use of robotic devices, may be beneficial. Imaging techniques focusing on assessments of collateral circulation and cerebral haemodynamics may help to select patients most likely to gain from IVT and MT, even beyond the timeframes investigated to date. The future may also hold promise of new thrombolytic agents and reperfusion techniques with greater efficacy and safety outcomes. Until more data are available, it is reasonable that all eligible patients should receive IVT before MT.

Minimising symptom onset to treatment time to facilitate early reperfusion is an important strategy to optimise patient outcome. Developing protocols both outside and inside stroke units is crucial in this regard. Mobile stroke units may play a role and it has been suggested that, in the future, they might also allow the use of novel diagnostic options (eg biomarkers and automated imaging evaluation) and therapies (eg neuroprotective drugs) in the prehospital setting. In rural and remote areas, the challenges of early stroke care are heightened and the use of specially designed aircrafts equipped with the ability to diagnose and treat acute stroke at remote emergency sites has been considered. ${ }^{75}$ We anticipate further major advances in acute interventions embedded within stroke units which will hopefully improve the quality and outcomes for acute stroke patients.

\section{Conflicts of interest}

Dr Mehool Patel is on the editorial board of Clinical Medicine.

\section{References}

1 Adeoye O, Nyström KV, Yavagal DR et al. Recommendations for the establishment of stroke systems of care: A 2019 update. Stroke 2019;50:e187-210

2 Kwan J, Hand P. Early neurological deterioration in acute stroke: clinical characteristics and impact on outcome. QJ Med 2006:99:635-33.

3 Mokin M, Priianini C, Siddiqui A, Turk AS. ASPECTS (Alberta Stroke Program Early CT Score) measurement using Hounsfield unit values when selecting patients for stroke thrombectomy. Stroke 2017:48:1574-9.

4 Rubiera M, Garcia Tornel A, Olive-Gadea M et al. Computed tomography perfusion after thrombectomy. An immediate surrogate marker of outcome after recanalization in acute stroke. Stroke 2020;51:1736-42.

5 Menon BK, d'Esterre CD, Qazi EM et al. Multiphase ct angiography: A new tool for the imaging triage of patients with acute ischemic stroke. Radiology 2015;275:510-20.

6 Laughlin B, Cahn A, Tai WA, Moftakhar P. RAPID automated CT perfusion in clinical practice. Pract Neurol 2019;2019:41-55.

7 Demeestere J, Wouters A, Christensen S, Lemmens R, Lansberg M. Review of perfusion imaging in acute ischemic stroke: from time to tissue. Stroke 2020;51:1017-24.

8 Langhorne P, Ramachandra S. Organised inpatient (stroke unit) care for stroke: network meta-analysis. Cochrane Database Syst Rev 2020;4:CD000197.

9 Ronning OM, Guldvog B. Stroke unit versus medical wards, II: Neurological deficits and activities of daily living. A quasi randomised controlled trial. Stroke 1998;29:586-90.

10 Inredavik B, Bakke RPT, Slordahl SA, Rokseth R, Haheim LL. Treatment in a combined acute and rehabilitation stroke unit. Which aspects are most important? Stroke 1999;30:917-23.

11 Middleton S, McElduff P, Ward J, Grimshaw JM et al. Implementation of evidence-based treatment protocols to manage fever, hyperglycaemia, and swallowing dysfunction in acute stroke (QASC): a cluster randomised controlled trial. Lancet 2011:378:1699-706.

12 Bhalla A, Wolfe CDA, Rudd AG. Management of acute physiological parameters after stroke. QJM 2001;94:167-72. 
13 Intercollegiate Stroke Working Party. National Clinical Guideline for Stroke, 5th Edition. Royal College of Physicians, 2016.

14 Powers WJ, Rabinstein AA, Teri Ackerson T et al. Guidelines for the early management of patients with acute ischemic stroke: 2019 update to the 2018 guidelines for the early management of acute ischemic stroke. Stroke 2019;50:e344-e418.

15 Roffe C, Ali K, Warusevitane A, Sills S et al. The SOS pilot study: a RCT of routine oxygen supplementation early after acute strokeeffect on recovery of neurological function at one week. PLoS One 2011;6:e19113.

16 Gray CS, Hildreth AJ, Sandercock PA et al. Glucose-potassiuminsulin infusions in the management of post-stroke hyperglycaemia: the UK Glucose Insulin in Stroke Trial (GIST-UK) 16. Lancet Neurol 2007:6:397-406.

17 CLOTS Trials Collaboration. Effect of intermittent pneumatic compression on disability, living circumstances, quality of life, and hospital costs after stroke: secondary analyses from CLOTS 3, a randomised trial. Lancet Neurol 2014;13:1186-92.

18 Geeganage CM, Sprigg N, Bath MW, Bath PMW. Balance of symptomatic pulmonary embolism and symptomatic intracerebral hemorrhage with low-dose anticoagulation in recent ischemic stroke: a systematic review and meta-analysis of randomized controlled trials. J Stroke Cerebrovasc Dis 2013;22:1018-27.

19 Whiteley WN, Adams HP, Bath PMW, Berge E et al. Targeted use of heparin, heparinoids, or low-molecular-weight heparin to improve outcome after acute ischaemic stroke: An individual patient data meta-analysis of randomised controlled trials. Lancet Neurol 2013;12:539-45.

20 Dennis M, Sandercock P, Reid J et al. The effect of graduated compression stockings on long-term outcomes after stroke: the CLOTS trials 1 and 2. Stroke 2013;44:1075-9.

21 Anderson CS, Arima H, Lavados $\mathrm{H}$ et al. Cluster randomised, crossover trial of head positioning in acute stroke. N Engl J Med 2017:376:2437-47.

22 Middleton S, McElduff P, Ward ] et al. Implementation of evidence-based treatment protocols to manage fever, hyperglycaemia, and swallowing dysfunction in acute stroke (QASC): a cluster randomised controlled trial. Lancet 2011, 378:1699-706.

23 Carlberg B, Asplund K, Hägg E. Factors influencing admission blood pressure levels in patients with acute stroke. Stroke 1991;22: 527-530.

24 ENOS Trial Investigators. Efficacy of nitric oxide, with or without continuing antihypertensive treatment, for management of high blood pressure in acute stroke (ENOS): a partial-factorial randomised controlled trial. Lancet 2015;385:617-28.

25 RIGHT-2 Investigators. Prehospital transdermal glyceryl trinitrate in patients with ultra-acute presumed stroke (RIGHT-2): an ambulance-based, randomised, sham-controlled, blinded, phase 3 trial. Lancet 2019;393:1009-20.

26 He J, Zhang Y, Xu T et al. Effects of immediate blood pressure reduction on death and major disability in patients with acute ischemic stroke: the CATIS randomized clinical trial. JAMA 2014:311:479-89.

27 Anderson CS, Huang Y, Lindley RI et al. Intensive blood pressure reduction with intravenous thrombolysis therapy for acute ischaemic stroke (ENCHANTED): an international, randomised, open-label, blinded-endpoint, phase 3 trial. Lancet 2019;393: 877-88.

28 De Havenon A, Bennett A, Stoddard G et al. Determinants of the impact of blood pressure variability on neurological outcome after acute ischaemic stroke. Stroke Vasc Neurol 2017;2:e000057.

29 Bath PMW, Krishnan K. Interventions for deliberately altering blood pressure in acute stroke. Cochrane Database Syst Rev 2014;10:CD000039.

30 Emberson J, Lees KR, Lyden P et al. Effect of treatment delay, age, and stroke severity on the effects of intravenous thrombolysis with alteplase for acute ischaemic stroke: A meta-analysis of individual patient data from randomised trials. Lancet 2014;384:1929-35.

31 Khatri P, Kleindorfer D, Devlin T et al. Effects of alteplase versus aspirin on functional outcome for patients with acute ischaemic stroke and minor nondisabling neurological deficits. The PRISMS Randomised Controlled Trial. JAMA 2018:320:156-66.

32 Fink JN, Kumar S, Horkan C et al. The stroke patient who woke up: clinical and radiological features, including diffusion and perfusion MRI. Stroke 2002;33:988-93.

33 Rimmele D, Thomalla G. Wake up stroke; clinical characteristics, imaging findings, and treatment option: an update. Front Neurol 2014:5:35.

34 Thomalla G, Simonsen CZ, Boutite F et al. MRI Guided thrombolysis for stroke with unknown time of onset. N Eng J Med 2018;379:611-22.

35 Campbell BCV, Ma H, Ringleb PA et al. Extending thrombolysis to 4.5-9 h and wake-up stroke using perfusion imaging: a systematic review and meta-analysis of individual patient data. Lancet 2019:394;139-47

36 Thomalla G, Boutitie F, Ma H et al. Intravenous alteplase for stroke with unknown time of onset guided by advanced imaging; systematic review and meta-analysis of individual patient data. Lancet 2020:6736:32163-2.

37 Genentech. Tenecteplase in stroke patients between 4.5 and 24 Hours (TIMELESS) (NCT03785678). https://clinicaltrials.gov/ct2/ show/NCT03785678.

38 Burgos AM, Saver JL. Evidence that tenecteplase is noninferior to alteplase for acute ischemic stroke meta-analysis of 5 randomized trials. Stroke 2019;50:2156-62.

39 Campbell BCV, Mitchell PJ, Churilov L et al. Tenecteplase versus alteplase before thrombectomy for ischemic stroke. N Engl J Med 2018;378:1573-82.

40 Coutts SB, Berge E, Campbell BC, Muir KW, Parsons MW. Tenecteplase for the treatment of acute ischemic stroke: A review of completed and ongoing randomized controlled trials. Int ] Stroke 2018;13:885-92.

41 Phipps MS, Cronin CA. Management of acute ischaemic stroke. BMJ 2020; 368:16983.

42 Campbell BCV. Clot length assessment in stroke therapy decisions. Stroke 2016;47:643-4.

43 Bray BD, Campbell J, Cloud GC et al. Bigger, faster? Associations between hospital thrombolysis volume and speed of thrombolysis administration in acute ischemic stroke. Stroke 2013;44:3129-35.

44 Kidwell C, Jahan R, Gornbein J et al. A trial of imaging selection and endovascular treatment for ischaemic stroke. N Eng J Med 2013;368:914-23.

45 Broderick J, Palesch Y, Demchuk A et al. Endovascular therapy with intravenous t-PA versus t-PA for stroke. N Eng J Med 2013;368: 893-9.

46 Goyal M, Menon BK, van Zwam WH et al. Endovascular thrombectomy after large-vessel ischaemic stroke: A meta-analysis of individual patient data from five randomised trials. Lancet 2016;387: 1723-31.

47 Berkhemer OA, Fransen PS, Beumer D et al. A randomized trial of intraarterial treatment for acute ischemic stroke. N Eng J Med 2015;372:11-20.

48 Muir KW, Ford GA, Messow CM et al. Endovascular therapy for acute ischaemic stroke: The pragmatic ischaemic stroke thrombectomy evaluation (PISTE) randomised, controlled trial. J Neurol Neurosurg Psychiatry 2017;88:38-44.

49 Goyal M, Demchuk AM, Menon BK et al. Randomized assessment of rapid endovascular treatment of ischemic stroke. N Eng J Med 2015;372:1019-30.

50 Campbell BC, Mitchell PJ, Kleinig T], Dewey HM, Churilov L, Yassi $\mathrm{N}$, et al. Endovascular therapy for ischemic stroke with perfusionimaging selection. N Eng J Med 2015;372:1009-18. 
51 Saver JL, Goyal M, Bonafe A et al. Stent-retriever thrombectomy after intravenous t-pa vs. T-pa alone in stroke. N Eng J Med 2015;372:2285-95.

52 Bracard S, Ducrocq X, Mas JL et al. Mechanical thrombectomy after intravenous alteplase versus alteplase alone after stroke (thrace): A randomised controlled trial. Lancet Neurol 2016;15:1138-47.

53 Mocco ], Zaiday OO, von Kummer R et al. Aspiration thrombectomy after intravenous alteplase versus intravenous alteplase alone. Stroke 2016;47:2331-8.

54 Jovin TG, Chamorro A, Cobo E et al. Thrombectomy within 8 hours after symptom onset in ischemic stroke. N Eng J Med 2015;372:2296-306.

55 Khoury NN, Darsaut TE, Ghostine ] et al. Endovascular thrombectomy and medical therapy versus medical therapy alone in acute stroke: A randomized care trial. J Neuroradiol 2017;44:198-202.

56 Nogueira RG, Jadhav AP, Haussen DC et al. Thrombectomy 6 to 24 hours after stroke with a mismatch between deficit and infarct. N Eng J Med 2018:378:11-21.

57 Bourcier R, Goyal M, Liebeskind DS et al. Association of time from stroke onset to groin puncture with quality of reperfusion after mechanical thrombectomy: a meta-analysis of individual patient data from 7 randomized clinical trials. JAMA Neurol 2019;76:405-11.

58 National Institute for Health and Care Excellence. Stroke and transient ischaemic attack in over 16s: Diagnosis and initial management (NG128). Pharmacological treatments and thrombectomy for people with acute stroke. NICE, 2019. www.nice.org.uk/guidance/NG128.

59 Albers GW, Marks MP, Kemp S et al. Thrombectomy for stroke at 6 to 16 hours with selection by perfusion imaging. N Engl J Med 2018;378:708-18.

60 Menon BK, Hill MD, Davalos A et al. Efficacy of endovascular thrombectomy in patients with $\mathrm{M} 2$ segment middle cerebral artery occlusions: meta-analysis of data from the HERMES Collaboration. J Neurointerv Surg 2019;11:1065-9.

61 Forman R. ESO-WSO large clinical trials webinar: BASICS. American Stroke Association, 2020. https://journals.heart.org/bloggingstroke/2020/05/14/eso-wso-large-clinical-trials-webinar-basics/.

62 Ford G, James M, White P. Mechanical thrombectomy for acute ischaemic stroke: An implementation guide for the UK. Oxford Academic Health Science Network, 2019. www.oxfordahsn.org/ourwork/adopting-innovation/mt-guide

63 Aronsson M, Persson J, Blomstrand C, Wester P, Levin LA. Costeffectiveness of endovascular thrombectomy in patients with acute ischemic stroke. Neurology 2016;86:1053-9.
64 Allen M, Pearn K, James M et al. Maximising access to thrombectomy services for stroke in england: A modelling study. Eur Stroke J 2019:4:39-49.

65 Lenthall R, McConachie N, White P, Clifton A, Rowland-Hill C. BSNR training guidance for mechanical thrombectomy. Clin Radiol 2017:72:175.e11-175.e18.

66 Yang $P$, Zhang $Y$, Zhang $L$ et al. Endovascular thrombectomy with or without intravenous alteplase in acute stroke. N Engl J Med 2020;382:1981-93.

67 Menon BK, d'Esterre CD, Qazi EM et al. Multiphase CT angiography: A new tool for the imaging triage of patients with acute ischemic stroke. Radiology 2015;275:510-20.

68 Wan TF, Xu R, Zhao ZA, Lv Y, Chen HS, Liu L. Outcomes of general anesthesia versus conscious sedation for Stroke undergoing endovascular treatment: a meta-analysis. BMC Anesthesiol 2019;19:69.

69 Turk AS, Siddiqui A, Fifi JT et al. Aspiration thrombectomy versus stent retriever thrombectomy as first-line approach for large vessel occlusion (COMPASS): a multicentre, randomised, open label, blinded outcome, non-inferiority trial. Lancet 2019;393:998-1008.

70 Vahedi K, Hofmeijer J, Juettler E et al. Early decompressive surgery in malignant infarction of the middle cerebral artery: a pooled analysis of three randomised controlled trials. Lancet Neurol 2007:6:215-22.

71 Alexander P, Heels-Ansdell D, Siemieniuk R et al. Hemicraniectomy versus medical treatment with large MCA infarct: a review and meta-analysis. BMJ Open 2016;6:e014390.

72 Cho DY, Chen TC, Lee HC. Ultra-early decompressive craniectomy for malignant middle cerebral artery infraction. Surg Neurol 2003:60:227-32.

73 Jüttler E, Unterberg A, Woitzik J et al. Hemicraniectomy in older patients with extensive middle-cerebral-artery stroke. N Engl J Med 2014;370:1091-100.

74 Silke W, Zhao H, Easton D et al. Air-Mobile Stroke Unit for access to stroke treatment in rural regions. Int J Stroke 2018;13:568-75.

75 Ayling OGS, Alotaibi NM, Wang JZ et al. Suboccipital decompressive craniectomy for cerebellar infarction: a systematic review and meta-analysis. World Neurosurg 2018;110:450-9.

Address for correspondence: Dr Ajay Bhalla, St Thomas' Hospital, Westminster Bridge Road, South Bank, London SE1 7EH, UK.

Email: ajay.bhalla@gstt.nhs.uk 